\title{
Stability of a slab collisional microtearing mode
}

\author{
M. Hamed, ${ }^{1,2}$ M. Muraglia, ${ }^{2}$ Y. Camenen, ${ }^{2}$ and X. Garbet ${ }^{1}$ \\ ${ }^{1}$ CEA, IRFM F-13108 St-Paul-Lez-Durance, France \\ ${ }^{2}$ Aix-Marseille Université, CNRS, PIIM UMR 7345, Marseille, France
}

Correspondence: myriam.hamed@cea.fr

\begin{abstract}
Summary
The stability of a collisional microtearing (MTM) is investigated by means of linear gyro-kinetic simulations and a theoretical linear dispersion relation. The electric potential fluctuations and the magnetic curvature are neglected in the simulations to match the theoretical model assumptions. In these conditions, the stability domain, the mode growth rate and the mode frequency predicted by the theory and the simulations are in satisfactory agreement. The radial structure of the mode has been also investigated and the localisation of the parallel current around the resonnant surface predicted by the theory is recovered by the simulations.
\end{abstract}

Keywords: microtearing mode, electron heat transport, pedestal

\section{Introduction}

In tokamaks, confinement is a key issue to achieve fusion reactions. However, micro scale turbulence leads to radial transport and degrades the confinement. This is why high confinement regimes, called H-modes, are needed. H-mode are achieved by exceeding a threshold in heating power and characterized by the formation of "a pedestal" which is a narrow insulating region just inside the last closed flux surface. In the pedestal, the radial turbulent transport is reduced and a steep pressure gradient develops. The modelisation of the pedestal dynamics is an important question to predict temperature and density profiles in the tokamak edge. The model "EPED" 1, based on the stability of large scales MagnetoHydroDynamic (MHD) modes, is the most commonly used to characterize the pedestal region. However, several open questions remain, in particular the origin of electron heat transport at the pedestal top. Recent gyrokinetic simulations suggest that ion Larmor radius scale modes called Microtearing Modes (MTMs) are unstable in the core of conventional aspect ratio tokamaks 203, spherical tokamaks 45 and reversed field pinches 6, could be at the origin of the electron residual turbulence at the top of the pedestal and as a consequence, determine the pedestal width 378 . Microtearing modes belong to a class of instability where a modification of the magnetic field line topology is induced at the ion Larmor radius scale. This leads to the formation of magnetic islands, which can enhance the electron heat transport 9 . Although the stability of MTMs has been theoretically studied in the past 1013 , the destabilization mechanism at play in the pedestal region is not yet well understood owing to the number of candidates (electron temperature gradient, magnetic field curvature, magnetic shear, ...) that can drive a current sheet and lead to the growth of a magnetic island. This lack of understanding leads to an apparent disagreement between the linear theory and gyrokinetic simulations. Indeed, past linear theories show that a slab current sheet is stable in the absence of collisions 1011 whereas, in collisionless regimes, recent gyrokinetic simulations in toroidal geometry found unstable MTMs 2 . In this paper we attempt to reconcile the slab current sheet theory and gyrokinetic simulations, by downgrading the gyrokinetic simulations to keep only the physical ingredients present in the simple slab model. In section 2, we first derive the marginal stability of a slab collisional MTM in a kinetic framework including only the electron temperature gradient. In section 3, the theoretical mode growth rate and frequency are then compared to linear simulations performed with the gyrokinetic code GKW 14 . The radial structure of the MTM obtained in the simulations is also compared to the theory assumption. Finally, section 4 is devoted to a summary and a discussion of our results. 


\section{Marginal kinetic stability of a slab collisional microtearing mode}

Following 11, we investigate the stability of a slab current sheet around a resonant surface of radial position $r_{0}$. The magnetic perturbations are expressed as a function of the vector potential along the magnetic field line $\delta B_{x}=i k_{\theta} \delta A_{\|}$ and $\delta B_{\theta}=-\partial_{x} \delta A_{\|}$, with $x=r-r_{0}$ the radial distance to the resonant surface of the mode and $k_{\theta}$ the wave number in the poloidal direction. Neglecting the electric potential, we assume that the perturbed electron current along the magnetic field $\delta j_{\|}$is small outside $\left(\delta j_{\|, \text {outside }} \sim 0\right)$ the current layer while it is large inside and given by the Ampère's law

$$
\Delta \delta A_{\|}=\left(\partial_{x}^{2}-k_{\theta}^{2}\right) \delta A_{\|}=-\mu_{0} \delta j_{\|}
$$

As a consequence, the slope of the perturbed potentiel vector $\delta A_{\|}$is discontinuous accross the current sheet. The potential vector can be expressed as $\delta A_{\|}=\delta A_{\|, 0} e^{-\left|k_{\theta} x\right|}$ outside the layer. Inside the current sheet $\delta A_{\|}$is supposed to be constant. The matching between the two solutions is simply represented by the so-called $\Delta^{\prime}$ parameter 15 , which for a slab current sheet of width $\delta_{J}$ is defined as

$$
\Delta^{\prime}=\left.\frac{1}{\delta A_{\|_{0}}} \frac{d \delta A_{\|}}{d x}\right|_{-\delta_{J}} ^{\delta_{J}} \sim-2 k_{\theta}
$$

Integrating Eq. (1) over the current layer and using the expression of $\Delta^{\prime}$, one can derive the MTM dispersion relation from a kinetic description in the collisional regime. The perturbed electron current along the magnetic field line is obtained from the electron distribution function whose evolution is given by the Vlasov-Boltzman equation. The distribution function is expressed as $f=F_{e q}+\delta f$ with $F_{e q}$ a maxwellian and $\delta f$ the perturbed distribution function. The Vlasov-Boltzman equation is then linearized using the time invariance translation $\delta f=\tilde{f} e^{-i \omega t}+$ c.c.. The expression for the perturbed distribution function $\delta f$ can be obtained by a decompostion of $\tilde{f}$ into a series of Legendre polynomials in the pitch-angle variable, $\tilde{f}=f_{0}(v)+f_{1}(v) \xi$ where $\xi=v / v_{T h_{e}}$ and $v_{T h_{e}}=\sqrt{2 T_{e} / m_{e}}$ is the electron thermal velocity with $T_{e}$ and $m_{e}$ the electron temperature and the electron mass, respectively. $f_{0}$ has no pitch-angle dependence and does not contribute to the current contrary to $f_{1}$. Thus, for a model taking into account the electron temperature gradient and neglecting the magnetic field curvature and the electric field the expression for $f_{1}$ is:

$$
f_{1}=-\frac{e}{T_{e}} F_{e q} v \delta A_{\|} \frac{\omega-\omega^{*}}{\omega+i \nu-\frac{k_{\|}^{2} v^{2}}{3 \omega}}
$$

where $\omega^{*}=\omega_{T}^{*}\left(\frac{1}{\eta_{e}}+\xi^{2}-\frac{3}{2}\right)$ is the diamagnetic frequency with $\omega_{T}^{*}=\frac{k_{\theta} \rho_{i}}{2} \sqrt{\frac{m e}{m i}} \frac{v_{T h_{e}}}{R} \frac{R}{L_{T_{e}}}$ where $m_{i}$ and $\rho_{i}$ are, respectively, the ion mass and the ion Larmor radius, $R$ is the reference length and $\eta_{e}$ is the ratio of electron temperature scale length $L_{T_{e}}$ and electron density scale length $L_{n_{e}} \cdot \nu=\nu_{e i T}\left(v_{T h_{e}} / v_{\|}\right)^{3}$ is the collision frequency derived from the collision operator in the Vlasov-Boltzman equation. The wave-number along the magnetic field of the perturbation is $k_{\|}=\frac{\hat{s} k_{\theta}}{R q} x=k_{\|}^{\prime} x$ where $\hat{s}$ is the magnetic shear and $\mathrm{q}$ is the safety factor (near the rational surface $q=m / n$ where $\mathrm{m}$ and $\mathrm{n}$ are respectively the poloidal and the toroidal mode numbers). The integration in the radial direction of the Ampère's law leads to

$$
\frac{d_{e}^{2} \Delta^{\prime}}{\Delta_{J}}=-\frac{8}{3 \sqrt{\pi}} \int_{-\infty}^{+\infty} d \rho \int_{0}^{+\infty} \xi^{4} e^{-\xi^{2}} d \xi \frac{\hat{\Omega}-\frac{1}{\eta_{e}}+\frac{3}{2}-\xi^{2}}{\hat{\Omega}-\frac{\rho^{2} \xi^{2}}{3 \hat{\Omega}}+i \frac{\hat{\nu}_{e i}^{t h}}{\xi^{3}}},
$$

In Eq.(4), a number of normalizations have been introduced for convenience. The instability takes place inside the current layer whose width is defined as $\Delta_{J}=\frac{q}{\hat{S}} \frac{R}{L_{T}} k_{\theta} \rho_{e} \sqrt{\nu_{e i}^{t h}}$ where $\hat{\nu}_{e i}^{t h}=\nu_{e i T} / \omega_{T}^{*}$ is the electron-ion collision frequency normalized to $\omega_{T}^{*}$ and $d_{e}^{2}=\frac{m_{e}}{\mu_{0} N e^{2}}$ is the electron skin depth with $\mathrm{N}$ the local density. The mode complex frequency $\omega$ is decomposed into a real frequency part and a linear growth rate part, $\omega_{r}+i \gamma$, and normalized to $\omega_{T}^{*}$ : $\hat{\Omega}=\omega / \omega_{T}^{*}$. The radial direction is normalized to $\Delta_{J}: \rho=x / \Delta_{J}$. The integration over $\rho$ is given by Cauchy's residue theorem. Finally, the dispersion relation is

$$
\frac{1}{\beta^{*}}=i 8 \sqrt{\frac{\pi}{3}} \int_{0}^{+\infty} \xi^{9 / 2} e^{-\xi^{2}} d \xi \frac{\hat{\Omega}-\frac{1}{\eta_{e}}+\frac{3}{2}-\xi^{2}}{\left(\xi^{3}+i \frac{\hat{\nu}_{e i}^{t h}}{\hat{\Omega}}\right)^{1 / 2}} .
$$


Table 1: Input parameters used for the theoretical calculations and the linear simulations with GKW.

\begin{tabular}{cccccccc}
\hline $\mathbf{q}$ & $\mathbf{s}$ & $\beta(\%)$ & $R / L_{T e}$ & $R / L_{T i}$ & $R / L_{n e}$ & $R / L_{n i}$ & $k_{\theta} \rho_{i}$ \\
\hline 1.34 & 1.08 & 1.55 & 8 & 0 & 0.263 & 0.263 & 0.3 \\
\hline
\end{tabular}

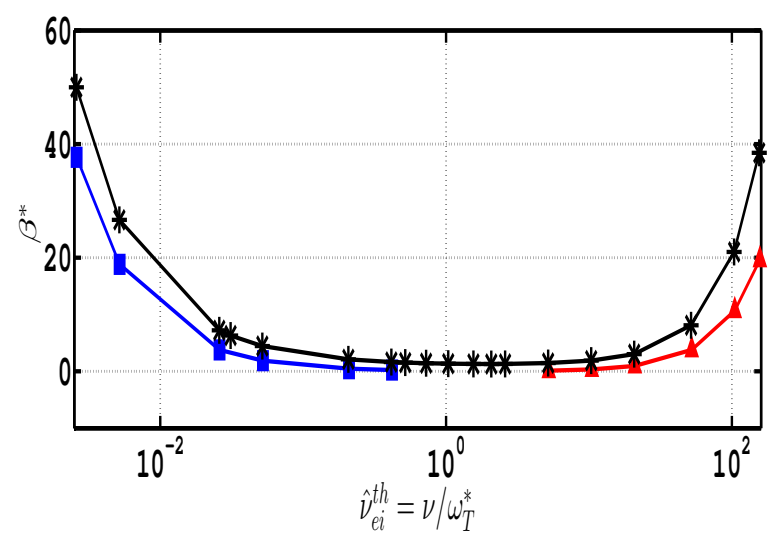

Figure 1: Marginal stability of a MTM as a function of $\hat{\nu}_{e i}^{t h}$ the electron collision frequency using the set of parameters presented in Table 1. Black cross line : marginal stability obtained solving Eq.(5), blue squares line : asymptotic limit $\hat{\nu}_{e i}^{t h} / \hat{\Omega}_{r} \rightarrow 0$, red triangle line : asymptotic limit $\hat{\nu}_{e i}^{t h} / \hat{\Omega}_{r} \rightarrow+\infty$.

where $\frac{1}{\beta^{*}}=\frac{d_{e}^{2} \Delta^{\prime}}{\Delta_{J}}$. By fixing the value of $\eta_{e}$ in Eq.5 it is possible to study the marginal stability of MTMs. For each value of $\hat{\nu}_{e i}^{t h}$, we determine the values of $\hat{\Omega}_{r}$ and $\beta^{*}$ solution of this equation. In Fig 1 , the parameter $\beta^{*}$ solution of Eq. 5 is shown as a function of the electron-ion collision frequency for each input parameters listed in Table 1 . Above the black cross line, MTMs are unstable and below MTMs are stable. The value of $\beta^{*}$ is related to plasma parameters by $\beta^{*}=\frac{\beta}{\left|k_{\theta} \rho_{e}\right|} \frac{R}{L_{T}} \frac{q}{\hat{s}}$ where $\beta$ is the ratio of the kinetic and magnetic pressures and $\rho_{e}=\sqrt{m_{e} / m_{i}} \rho_{i}$ is the electron Larmor radius. At low and high collisionality the MTM destabilization is only possible for strong electron temperature gradient and for high $\beta$. At intermediate collisionality MTMs are easier to destabilize. In the collisionless regime $\left(\hat{\nu}_{e i}^{t h} / \hat{\Omega}_{r} \rightarrow 0\right)$ and in the strongly collisional regime $\left(\hat{\nu}_{e i}^{t h} / \hat{\Omega}_{r} \rightarrow+\infty\right)$ Eq. 5 can be solved analytically. Thus, when $\hat{\nu}_{e i}^{t h} / \hat{\Omega}_{r} \rightarrow 0$ :

$$
\hat{\Omega}_{r}=\frac{1}{\eta_{e}}+\frac{1}{2} \quad \text { and } \quad \frac{1}{\beta *}=\sqrt{3} \pi \frac{\hat{\nu}_{e i}^{t h}}{\hat{\Omega}_{r}} .
$$

And when $\hat{\nu}_{e i}^{t h} / \hat{\Omega}_{r} \rightarrow+\infty:$

$$
\hat{\Omega}_{r}=\frac{1}{\eta_{e}}+\frac{5}{4} \quad \text { and } \quad \frac{1}{\beta *}=\frac{23}{2} \sqrt{\frac{\pi}{6}} \Gamma\left(\frac{17}{4}\right)\left(\frac{\hat{\Omega}_{r}}{\hat{\nu}_{e i}^{t h}}\right)^{3 / 2} .
$$

On Fig.(1), the asymptotic limits given by Eq.(6) and Eq.(7) are represented by the blue squares line and the red triangle line, respectively. The full calculation converge towards the asymptotics limits for very low and very high value of the collisionality $\left(\nu_{e i}^{t h}<10^{-6}\right.$ and $\nu_{e i}^{t h}>10^{14}$ for less than $1 \%$ difference). MTM is stable in both the collisionless and strongly collisional regime consistently with 1011 .

\section{$3 \quad$ MTM destabilization and radial structure in a gyrokinetic framework}

For comparison with the linear theory of a slab MTM, gyrokinetic simulations have been performed using the GKW code 14. GKW is a flux-tube code that computes turbulent transport in tokamak plasmas. It solves linearly and non linearly the Vlasov-Maxwell system of equation that determines the evolution of the electron and ion distribution function, as formulated by the gyrokinetic model 16 . The input parameters chosen for this study are given in Table 1. In the linear simulations the electric potential and the magnetic field curvature are neglected, to match the assumptions of the linear theory. The ion kinetic response, the toroidal geometry and finite Larmor radius effects (gyroaverage) are however retained. Collisions are included with a linearised Fokker-Planck operator (pitchangle scattering only).

To compare the rotation frequency and the growth rate of MTMs with GKW simulations, the collision frequency $\hat{\nu}_{e i}^{t h}$ has been renormalised using GKW normalisation, $\nu_{e i}^{G K W}=\hat{\nu}_{e i}^{t h} \frac{k_{\theta} \rho_{i}}{2} \sqrt{\frac{m_{e}}{m_{i}}} \frac{R}{L_{T_{e}}}$. 

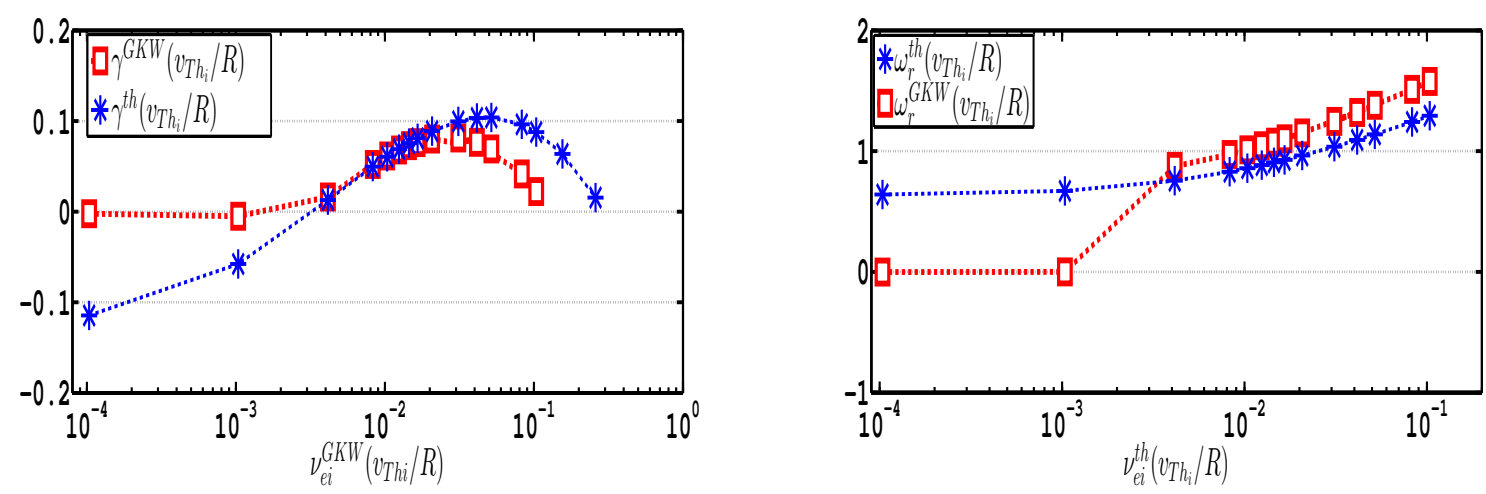

Figure 2: Left: Analytical and numerical linear growth rates as a function of electron collision frequency $\nu_{e i}^{G K W}$ for the set of parameters presented in Table 1. Right: Analytical and numerical real frequency as a function of $\nu_{e i}^{G K W}$.

In Fig. 2, a comparison between the linear kinetic theory Eq. (5) and the linear simulations is presented : analytical and numerical linear growth rates are shown as function of the electron collision frequency $\nu_{e i}^{G K W}$. As in 10 , MTMs are stable at low collisionality whereas they were unstable in simulations including more complex physical effects (curvature, electric potential...)2 2 . Then, at intermediate collisionality, MTMs are destabilized. The stability domain of the linear slab theory mostly matches that of the simulations. Even quantitatively, the agreement is satisfactory at intermediate collisionality, with differences starting to arise at low and high collisionalities. At high collisionality, the current layer width actually becomes wider which could challenge the "constant- $A_{\|}$" approximation made to formulate Eq. (5). The real frequency of the mode predicted by the simulations and the theory are also in reasonable agreement.

The radial structure of the mode is then investigated. In section 1 , the radial structure of the mode was assumed to be $\delta A_{\|, 0} e^{-\left|k_{\theta} x\right|}$ outside the resistive layer and $\delta A_{\|}=$cte inside. In the analytical calculation the mode structure is expressed in toroidal coordinates with $\delta A_{\|}(r, \theta, \varphi)=\sum_{m, n} \delta A_{\|, m, n}(r) e^{-i(m \theta+n \varphi)}$ where $r$ is the radial coordinate, $\theta$ is the poloidal angle and $\varphi$ is the toroidal angle. In GKW, field aligned Hamada coordinates 17 , $\psi, \zeta, s)$ are used, where $\psi$ and $\zeta$ are a flux label, $i . e . \mathbf{B} \cdot \nabla \psi=0$ and $\mathbf{B} \cdot \nabla \zeta=0$ and $s$ follows the magnetic field lines. In circular geometry $18 s=[\theta+\epsilon \sin \theta] / 2 \pi$ with $\epsilon$ the aspect ratio. In the code, $\delta A_{\|}$is represented by a sum over Fourier $\operatorname{modes} \delta A_{\|}(\psi, \zeta, s)=\sum_{k_{\zeta} k_{\psi}} \delta \hat{A}_{\|}\left(k_{\zeta}, k_{\psi}, s\right) e^{i k_{\zeta} \xi+i k_{\psi} \psi}$. The two representations are linked by:

$$
\delta A_{\|_{m_{0}, n}}(x)=e^{i k_{\epsilon}^{0} x} \int_{-\infty}^{+\infty} \frac{d \eta}{2 \pi} \delta \hat{A}_{\|}\left(k_{\epsilon}^{0}, k_{\zeta}, \eta\right) e^{i \frac{k_{\zeta}}{2 \pi} \frac{q_{0}}{\epsilon_{0}} \hat{s} x \eta}
$$

where $m_{0}=-n q$ and $\eta$ is the straight field line angle $\left(q=\frac{B \cdot \nabla \varphi}{B \cdot \nabla \eta}\right)$, related to GKW cordinates by: $\eta=\frac{R}{q} \frac{B_{t}}{B^{s}} \int_{0}^{s} \frac{d s}{R^{2}}$. Thus, the radial structure of the mode can be obtained by the inverse Fourier transform of the numerical eigenfunction $\delta A_{\|}(s)$. Fig, 3 presents the real and the complex part of the eigenfunction for linear simulations using the set of parameters presented in Table 1 at intermediate collisionality, $\nu_{e i}^{G K W}=0.02$. As expected the structure of the mode presents a tearing parity (i.e. $\delta A_{\|}$is even in the radial direction) allowing an island formation at ion Larmor radius scales. Moreover, the radial structure expected by the theory is recovered numerically, i.e. outside the resistive layer $\delta A_{\|}$decays as $e^{-\left|k_{\theta} x\right|}$. From Eq. 2 2), $\Delta^{\prime}$ is assumed to be equal to $-2 k_{\theta}$. This value is recovered when using the radial structure of the mode from GKW to compute $\Delta^{\prime}$. Finally, in Fig 4 , the theoretical current layer width (calculated at the $1 / 3$ maximum of the parallel current) is compared to the numerical value as a function of the collision frequency $\nu_{e i}^{G K W}$. Note that the current layer is very thin and localized around the resonant surface, Fig 3 . To describe correctly the current layer and to determine its width a high resolution is, therefore, necessary. The grid size is chosen to have 30 points in the current layer (399 poloidal turns and 31 points per poloidal turn). Fig. 5 shows the current layer width (up) and the growth rate (down) as a function of the poloidal turn $\left(n_{\text {pol }}\right)$ for $\nu_{\text {ei }}^{G K W}=0.02$. The growth rate is not impacted by the resolution scan but an accurate evolution of the current layer width require high resolution. 

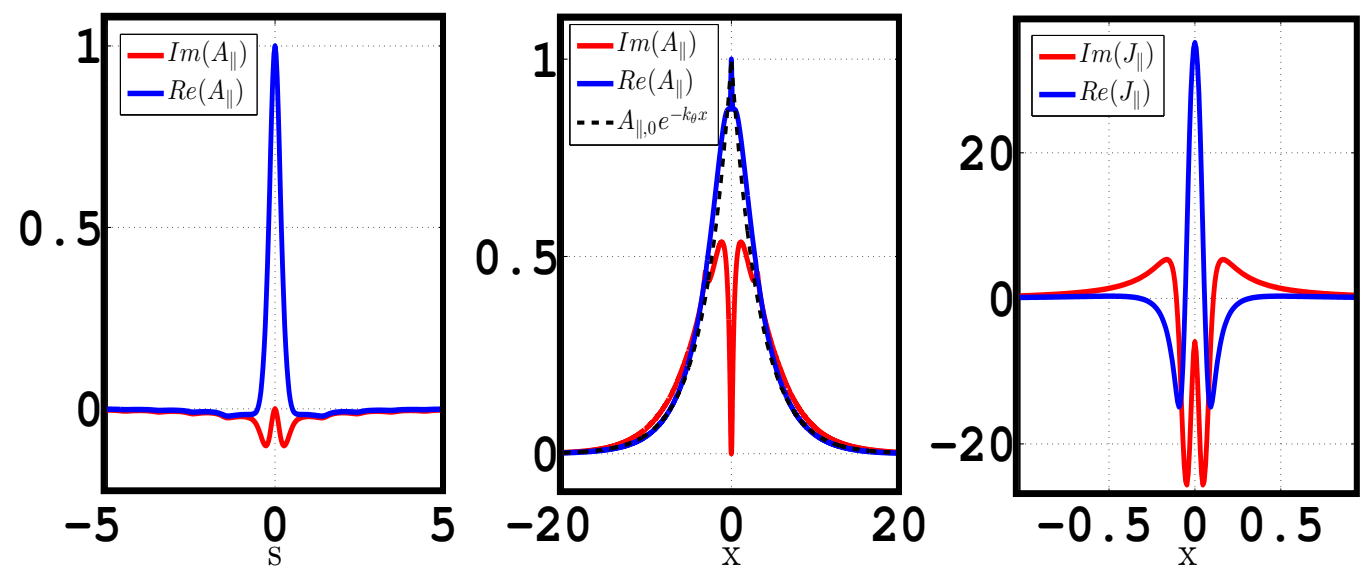

Figure 3: Real and complex part of the numerical eigenfunctions of MTMs in intermediate collisional regime (i.e. $\left.\nu_{e i}^{G K W}\left(v_{T h i} / R_{r e f}\right)=0.02\right)$. The left figure represent the mode structure in the parallel direction of the magnetic field line $\delta A_{\|}(s)$, the middle and the right figures represent respectively the radial structure of a MTM and the radial current profil calculated using the second derivative of $\delta A_{\|}(x)$. The eigenfunctions are rotated in the complex plane to have $\delta A_{\|}(s=0)=1$ and $\delta A_{\|}(x=0)=1$

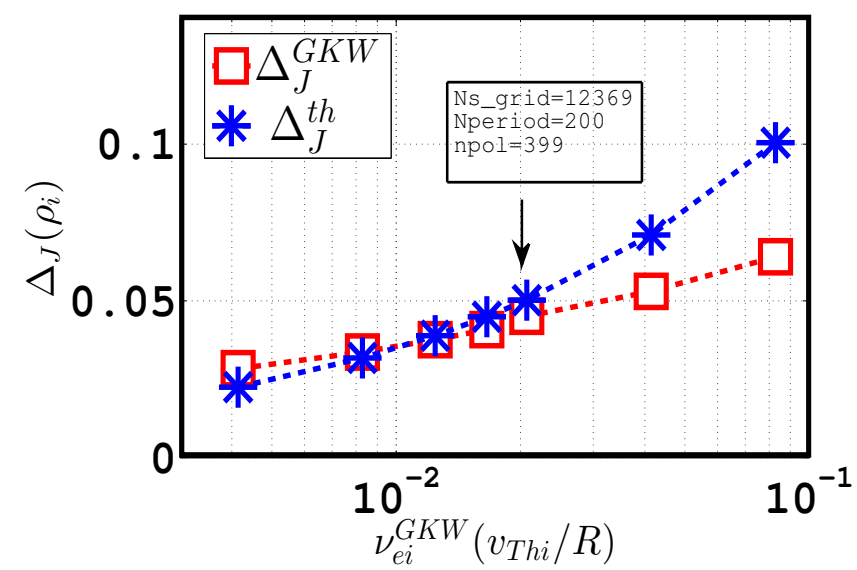

Figure 4: Comparison between the theoretical calculation of the current sheet width using the set of parameters presented in Table 1 and the numerical simulations as a function of the collisionnality frequency.

\section{Conclusions}

In conclusion, we have formulated a linear theory of the slab microtearing mode in the collisional regime using a kinetic approach. Its appears that both low and high collisionalities are stabilizing. We confirmed that slab MTMs are destabilized by the electron temperature gradient. A comparison of the theory with linear gyro-kinetic simulations has been performed. Provided the simulations neglect the electric potential and magnetic field curvature, as in the model, the gyro-kinetic simulations do recover the analytical linear theory. Moreover, we have compared the radial structure of the vector potential assumed by the theory with that obtained from numerical simulations. We have found a qualitative good agreement between the two structures. In particular, the parallel current is localized within a narrow layer. The current layer width predicted by the theory is also recovered by the numerical simulations. The linear stability of the collisionless MTMs predicted by 1011 is therefore consistent with numerical simulations. When the simulations include the curvature and electric potential MTM can be destabilised in the collisionless regime 28. 
Thus, adding step by step these terms in the theory, which will be the purpose of the future work, one should identify the different mechanisms at play in the MTM destabilization.

\section{Acknowledgments}

The authors thank C.M. Roach for fruitful discussions. This work was granted access to the HPC resources of AixMarseille Université financed by the project Equip@Meso (ANR-10-EQPX-29-01) of the program 'Investissements d'Avenir' supervised by the Agence Nationale pour la Recherche.

\section{References}

[1] P.B. Snyder, T.H. Osborne, K.H. Burrel, Phys. Plasmas 19, 056115 (2012).

[2] H. Doerk, F. Jenko, T. Görler, D.Told, M.J. Pueschel, and D.R. Hatch Phys. Plasmas 19, 055907 (2012).

[3] D.R. Hatch, M. Kotschenreuther et al., Nucl. Fusion 56, 104003 (2016).

[4] W. Guttenfelder, J. Candy, S.M. Kaye, W.M. Nevins, R.E. Bell et al., Phys. Plasmas 19, 022506 (2012).

[5] D.J. Applegate, C.M. Roach, J.W. Connor, S.C. Cowley, W. Dorland et al., Plasma Phys. Control. Fusion 49, 1113 (2007).

[6] I. Predebon, F. Sattin, M.Veranda, et al. Phys. Plasmas 20, 040701 (2013).

[7] S. Saarelma, M.N.A. Beurskens, T. Casper, et al., IAEA FEC conference San Diego (2012).

[8] D. Dickinson, C.M. Roach, S. Saarelma, R. Scannell, A. Kirk and H.R. Wilson, Phys. Rev. Let. 108, 135002 (2012).

[9] W. Guttenfelder, J. Candy, et al., Phys. Rev. Lett. 106, 155004 (2011).

[10] R. D. Hazeltine, D. Dobrott and T.S. Wang, Phys. Fluids 18, 1778 (1975).

[11] J.F. Drake and Y.C. Lee, Phys. Fluids 20, 1341 (1977).

[12] J.W. Connor, S.C. Cowley and R.J. Hastie, Plasma Phys. Control. Fusion 32, (1990).

[13] A.K. Swamy, R. Ganesh, J. Chowdhuri, S. Brunner, J. Vaclavik, and L. Villard, Phys. Plasmas 21, 082513 (2014).

[14] A.G. Peeters et al., Computer Physics Communication 180, 2650 (2009).

[15] A. Poyé, O. Agullo, A. Smolyakov, S. Benkadda and X. Garbet, Phys. Plasmas 20, 020702 (2013).

[16] A. Brizard, Phys. Plasmas 41, 541 (1988).

[17] S. Hamada, Kakuyugo Kenkyu 1, 542 (1958).

[18] X. Lapillonne et al., Phys. Plasmas 16, 032308 (2009). 
$\square$ 